\title{
Clinical efficacy of arthroscopy in the treatment of discoid meniscus injury and related risk factors for postoperative pain
}

\author{
Jiong Lu, Yinan Chen, Minwei Hu, Changhui Sun \\ Department of Orthopedic, Affiliated Ruijin Hospital Luwan branch, Shanghai Jiao Tong University School of Medicine, Shanghai, China \\ Contributions: (I) Conception and design: J Lu, C Sun; (II) Administrative support: Y Chen; (III) Provision of study materials and patients: J Lu, Y \\ Chen, M Hu; (IV) Collection and assembly of data: All authors; (V) Data analysis and interpretation: J Lu, M Hu, C Sun; (VI) Manuscript writing: \\ All authors; (VII) Final approval of manuscript: All authors. \\ Correspondence to: Changhui Sun. Department of Orthopedic, Affiliated Ruijin Hospital Luwan branch, Shanghai Jiaotong University School of \\ Medicine, No 149 Chongqing South Road, Huangpu District, Shanghai 200020, China. Email: steven_luke@163.com.
}

Background Discoid meniscus is an abnormal meniscus of the knee joint. This study aimed to analyze the clinical efficacy of arthroscopy in the treatment of discoid meniscus injury, and determine the related risk factors for postoperative pain.

Methods: A total of 80 patients with a discoid meniscus injury who were diagnosed and treated in our hospital from May 2017 to May 2018 were selected. According to the different surgical methods, they were divided into the study group (treated with meniscus plasty) or the control group (treated with subtotal meniscectomy). Knee joint motion and knee joint function were measured at 2, 6 and 12 weeks post-surgery. Knee joint function was measured using the Lysholm Knee Scoring Scale to determine clinical efficacy. Patients with an excellent and good rating were included in the painless group, and patients with an average and poor rating were included in the pain group. The risk factors for postoperative pain were analyzed using multivariate logistic regression.

Results: Knee joint motion in the study group and the control group gradually increased and reached a peak 12 weeks after surgery $(\mathrm{P}<0.05)$, however, there was no significant difference between the 2 groups at each time point after surgery $(\mathrm{P}>0.05)$. The excellent and good rate, according to the Lysholm Knee Scoring Scale, was $84.62 \%$ in the study group and $84.38 \%$ in the control group. There was no significant difference between the 2 groups $(\mathrm{P}>0.05)$. There were differences between the pain group and the painless group in Watanabe classification, age, preoperative symptom time, cold compress, joint soft tissue injury, and postoperative weight-bearing time $\leq 1$ week $(\mathrm{P}<0.05)$.

Conclusions: Arthroscopy demonstrated efficacy for the treatment of patients with a discoid meniscus injury. Age, duration of preoperative symptoms, articular cartilage injury, postoperative cold compress and postoperative weight-bearing time were independent risk factors that affected postoperative pain after arthroscopic surgery. For high-risk patients, effective prevention and control measures should be taken to improve postoperative pain and promote the recovery of knee joint function.

Keywords: Arthroscopy; discoid meniscus; pain; clinical efficacy; risk factors

Submitted Sep 03, 2020. Accepted for publication Nov 06, 2020.

doi: 10.21037/apm-20-1899

View this article at: http://dx.doi.org/10.21037/apm-20-1899 


\section{Introduction}

Discoid meniscus, also known as discoid cartilage, is an abnormal manifestation of the meniscus of the knee joint. A discoid meniscus usually does not cause any discomfort or other symptoms before the injury, with only a few patients showing knee joint bounce during knee flexion and other activities. However, when injured, the discoid meniscus will cause tenderness, bounce, and edema in the lateral joint space of the knee joint, and some patients may even experience interlocking (1). Surgical treatment is a necessary measure to relieve the clinical symptoms of discoid meniscus injury, recover the function of the knee joint, and avoid degeneration. However, because the traditional incision surgery has a lot of disadvantages such as significant damage, multiple complications, and slow recovery, arthroscopic treatment has become more popular in recent years (2). There are many studies on arthroscopic and discoid meniscus injury, but most of them focus on the treatment efficacy and prognosis evaluation. In addition, clinical practice findings show that some patients may suffer from different degrees of knee pain after surgery, which not only affects patients' postoperative rehabilitation exercises, but also causes a decline in their quality of life. Therefore, determining the risk factors that affect postoperative pain is the new hot spot for the timely prevention and treatment of discoid meniscus injury (3). Therefore, this study analyzed the clinical efficacy of arthroscopic treatment for discoid meniscus injury, and determined the related risk factors for postoperative pain, aiming to provide further insights into clinical treatment approaches for discoid meniscus injury. We present the following article in accordance with the STROBE reporting checklist (available at http://dx.doi. org/10.21037/apm-20-1899).

\section{Methods}

\section{Patients and study design}

This study was approved by the ethics committee of Affiliated Ruijin Hospital (No. 2019-129). A total of 80 patients with discoid meniscus injury of the knee joint who were diagnosed and treated in our hospital from May 2017 to May 2018 were selected. Among them, 3 cases were discontinued, 6 cases withdrew, leaving a total of 71 enrolled cases. All patients provided informed consent. According to the different surgical methods, they were divided into 2 groups: study group $(n=39)$ and control group $(n=32)$. In the study group, there were 24 males and 15 females, aged 28
70 years old, with an average age of $49.39 \pm 6.31$ years old; 11 cases had the injury in the left knee joint and 28 cases had the injury in the right knee joint. In the control group, there were 21 males and 11 females, aged 28-72 years old, with an average age of $49.53 \pm 6.42$ years old; 10 cases had the injury in the left knee joint and 22 cases had the injury in the right knee joint.

Patients were included in the study if they met the diagnostic criteria as per Practical Orthopedics (4) for discoid meniscus injury of the knee joint, and had no history of major surgery on the knee joint. The exclusion criteria were as follows: (I) patients with major organ injury or tumor; (II) patients with severe joint disease in multiple parts of the body; (III) patients with incomplete general clinical data; (IV) patients with severe mental illness that could not cooperate with the study. All procedures performed in this study involving human participants were in accordance with the Declaration of Helsinki (as revised in 2013).

\section{Surgical methods}

All patients underwent knee arthroscopy and microscopic treatment. Routine magnetic resonance imaging (MRI) examination was performed before the operation to confirm the meniscus injury. Patients in the control group underwent subtotal meniscectomy, and those in the study group underwent meniscus plasty.

The knee arthroscopy equipment used in the operation were from Smith \& Nephew Company (USA), with a wideangle $\left(30^{\circ}\right)$, high-definition camera imaging system and a $4 \mathrm{~mm}$ diameter. The patient was placed in a supine position, with the knee joint flexed at $90^{\circ}$. After routine disinfection, the patient underwent general anesthesia or epidural anesthesia under ECG monitoring. The anterior medial and lateral sides of the knee joint were selected as the surgical approaches for knee arthroscopy, and the overall status of the meniscus was observed under the microscope. For those with high surgical difficulty, the median patellar ligament was added as the surgical approach.

Meniscus plasty was performed as follows: after inserting the arthroscope, the anterior medial approach was chosen to insert the planing knife to clean the hyperplastic synovium. The anterolateral approach was then chosen to insert the blue forceps to remove the thickened tissue, and to trim the incision to keep the structure of the meniscus in a "C" shape. For patients with a lamellar tear, the next layer was excised, while for patients with a barrel-shaped tear, the 


\begin{tabular}{|c|c|c|c|c|c|}
\hline \multirow[t]{4}{*}{ Claudication (5 scores) } & No & 5 (scores) & Swell (10 scores) & No & 10 scores \\
\hline & Mildly or intermittently lameness & 3 & & After overexertion & 6 \\
\hline & Severe or persistent lameness & 0 & & After normal exertion & 2 \\
\hline & & & & Stay swollen & 0 \\
\hline \multirow[t]{4}{*}{ Support (5 scores) } & No & 5 & Upstairs [10] & No problem & 10 \\
\hline & With a cane or crutch & 2 & & Modestly attenuated & 6 \\
\hline & Can't load & 0 & & Difficult for every step & 2 \\
\hline & & & & Can't go upstairs & 0 \\
\hline \multirow[t]{5}{*}{ Twist locks } & No sense of twist lock or stuck & 15 & Squat [5] & No problem & 5 \\
\hline & Little twist lock and no stuck & 10 & & Modestly attenuated & 4 \\
\hline & Accidently & 6 & & Over $90^{\circ}$ & 2 \\
\hline & Frequently & 2 & & Can't squat & 0 \\
\hline & During medical examination & 0 & & & \\
\hline \multirow[t]{6}{*}{ Weakness (25 scores) } & Never & 25 & Pain [25] & Never & 25 \\
\hline & Occasionally (during exercise or exertion) & 20 & & Rare or mild when exertion & 20 \\
\hline & Frequently (during exercise or exertion) & 15 & & Significant when exertion & 15 \\
\hline & Occasionally (during daily life) & 10 & & Significant after $2 \mathrm{~km}$ walk & 10 \\
\hline & Frequently (during daily life) & 5 & & Significant after less than $2 \mathrm{~km}$ walk & 5 \\
\hline & Every step & 0 & & Continuous & 0 \\
\hline
\end{tabular}

Figure 1 Lysholm Knee Scoring Scale table used in this study.

operation was completed along the tear edge.

Subtotal meniscectomy was performed as follows: after inserting the arthroscope, the cold light source was turned on and the hyperplastic synovium was trimmed. The blue forceps were inserted from the anterior side to remove the severely damaged meniscus. For those who were severely torn, with the surrounding tissues affected, the soft tissue of the corresponding meniscus was removed, the debris was cleaned, and the joint cavity cleaning operation was completed at the same time.

After the operation was completed, the surgical incision was closed after removing the medical instruments such as the arthroscope from the articular cavity. After surgery, bandages were applied, and vital signs were continuously monitored. At the same time, patients were given an anticoagulant, analgesic, anti-inflammatory treatment and rehabilitation exercises.

\section{Observation indicators}

\section{Clinical efficacy}

At week 2, week 6, and week 12 post-surgery, the same group of nursing staff evaluated the function of the knee joint in the 2 groups of patients according to the Lysholm Knee Scoring Scale (see Figure 1 for details) (5). The scoring range was $0-100$ points, where scores $\geq 90$ points were excellent, 80-90 points were good, 60-80 points were average, and $<60$ points were bad. The excellent rate was calculated and patients with scores of excellent and good were included in the painless group, while patients with scores of average and poor were included in the pain group.

\section{The motion range of the knee}

The motion range of the knee was compared between the 2 groups at week 2, week 6 and week 12 after arthroscopy. The same group of nursing staff were arranged to measure the maximum degree of knee flexion of the affected limb.

\section{Risk factors}

Risk factors of postoperative pain were assessed including articular cartilage injury, surgical method, cold compress, postoperative weight bearing time, body mass index, age, gender, duration of preoperative symptoms, and Watanabe classification. 
Table 1 Comparison of the motion range of the knee joint between the 2 groups after surgery

\begin{tabular}{lcccccc}
\hline Group & $\mathrm{n}$ & Week 2 & Week 6 & Week 12 & $\mathrm{F}$ \\
\hline Study group & 39 & $57.96 \pm 8.13$ & $101.15 \pm 9.53$ & $127.84 \pm 6.64$ & 723.80 & $<0.001$ \\
Control group & 32 & $58.31 \pm 8.27$ & $102.23 \pm 9.75$ & $128.15 \pm 6.33$ & 569.54 & - \\
$t$ & & 0.179 & 0.470 & 0.200 & - & - \\
$\mathrm{P}$ & & 0.858 & 0.640 & 0.842 & - \\
\hline
\end{tabular}

Data are presented as $\bar{x} \pm \mathrm{s}$.

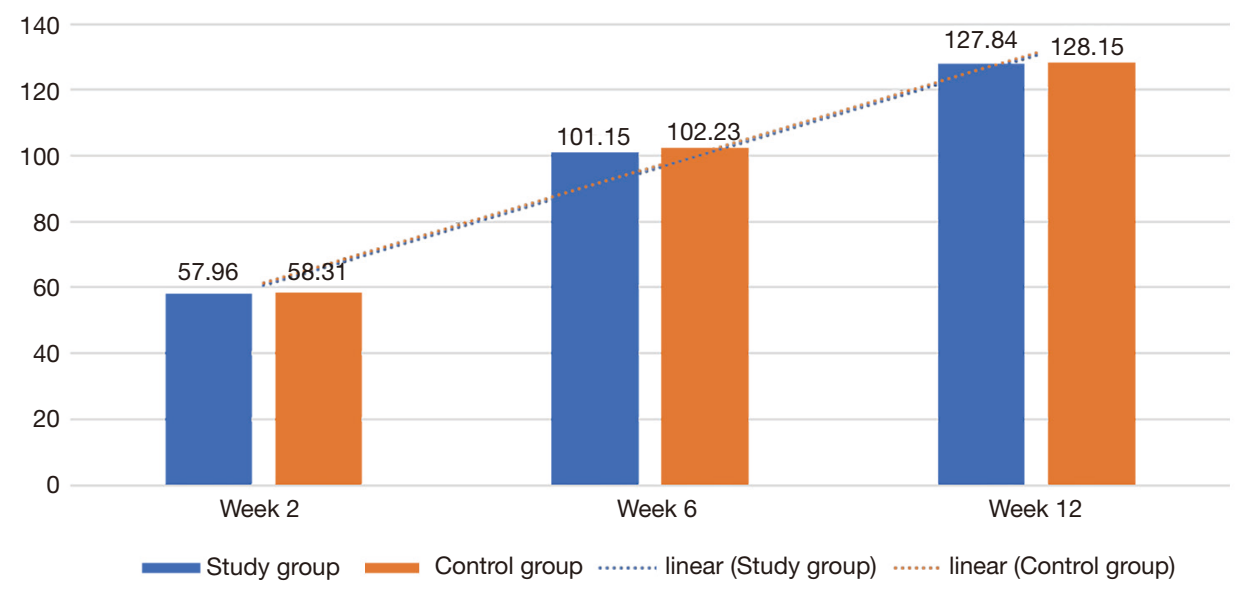

Figure 2 Comparison of the motion range of the knee joint between patients who underwent different surgical methods for the treatment of discoid meniscus. The motion range of the knee joint in the 2 groups was detected over time; however, there was no significant difference between the 2 groups for each time point after surgery.

\section{Statistical methods}

The data in this study were statistically analyzed using SPSS 22.0 software (IBM, USA). The measurement data were expressed as mean \pm standard deviation. Data such as clinical efficacy, age, gender, articular cartilage injury, surgical method, cold compress, Watanabe classification and other measurement data were analyzed by $\mathrm{F}$ test or $t$ test. The measurement data such as the motion of knee joint, postoperative weight-bearing time, body mass index, preoperative symptom duration was described as the mean \pm standard deviation and analyzed by $\chi^{2}$ test. Multivariate logistic regression analysis was used to analyze the risk factors affecting postoperative pain in patients with discoid meniscus injury. $\mathrm{P}<0.05$ was considered to be statistically significant.

\section{Results}

\section{Motion range of knee joint}

There were no significant differences in baseline characteristics between the 2 groups $(\mathrm{P}>0.05)$.

The motion range of the knee joint in the 2 groups gradually increased over time and reached a peak during week $12(\mathrm{P}<0.05)$, however, there was no significant difference between the 2 groups for each time point after surgery ( $\mathrm{P}>0.05$, Table 1 and Figure 2$)$.

\section{Clinical efficacy}

The excellent and good rates on the Lysholm Knee Scoring Scale were $84.62 \%$ in the study group and $84.38 \%$ in the 
Table 2 Comparison of clinical efficacy between the 2 groups as measured by the Lysholm Knee Scoring Scale

\begin{tabular}{lcccccc}
\hline Group & $\mathrm{n}$ & Excellent & Good & Average & Poor & Excellent and good rate \\
\hline Study group & 39 & $19(48.72)$ & $14(35.90)$ & $4(10.26)$ & $2(5.13)$ & 84.62 \\
Control group & 32 & $15(46.88)$ & $12(37.50)$ & $4(12.50)$ & $1(3.13)$ & 84.38 \\
$\chi^{2}$ & & 0.024 & 0.019 & 0.088 & 0.174 \\
$P$ & 0.877 & 0.889 & 0.766 & 0.676 & \\
\hline
\end{tabular}

Data are presented as $\mathrm{n}(\%)$.

control group, showing no significant difference between the 2 groups $(\mathrm{P}>0.05$, Table 2$)$.

\section{Single factor analysis of postoperative pain in patients with discoid meniscus injury of the knee joint}

Among the 71 enrolled patients, those with excellent and good postoperative efficacy were included in the painless group, and those with average and poor postoperative efficacy were included in the pain group $(n=11)$. There was no significant difference in gender, body mass index, and surgical method between the 2 groups $(\mathrm{P}>0.05)$. However, factors such as Watanabe classification, age, preoperative symptoms, preoperative symptom duration, cold compress, joint soft tissue injury and postoperative weight-bearing time $\leq 1$ week were significantly different between the 2 groups $(\mathrm{P}<0.05$, Table 3$)$.

\section{Multi-factor analysis of postoperative pain in patients with discoid meniscus injury of the knee joint}

Results indicated that factors such as age, preoperative symptom duration, postoperative weight-bearing time $\leq 1$ week, cold compress, and joint soft tissue injury were all independent risk factors that affected postoperative pain in patients with discoid meniscus injury $(\mathrm{P}<0.05$, Table 4).

\section{Discussion}

As an abnormal structure, discoid meniscus of the knee joint is characterized by thickening of the tissue (6). Because the thickened discoid meniscus changes the biomechanical characteristics of the knee joint, it is not conducive to the load transmission of the knee joint. It may cause concentrated pressure on the center of the discoid meniscus, and cause degeneration, injury, tearing, and cystic changes, which have a significant impact on patients (7). At the same time, due to the abnormal function and morphological changes of synovial membrane in the joint, excessive synovial fluid generation and absorption disorders may result in joint effusion with joint swelling, pain, limited function, muscle atrophy and other major clinical manifestations. Therefore, active treatment measures are required for patients with discoid meniscus injury.

Several clinical studies have shown that surgical treatment is the best therapy for discoid meniscus, as conservative medical treatment has no obvious effect (8). In the past, open surgery was the only solution to improve the shape and function of the meniscus. However, with the wide application of arthroscopy, treatment with minimal invasion has become the current mainstream treatment for discoid meniscus (9). Studies have shown that with arthroscopy, a clear diagnosis, small trauma, quick postoperative recovery, and low incidence of complications such as infection can be achieved (10). Arthroscopic treatment is mainly divided into subtotal meniscectomy and meniscus plasty. The results in Table 1 and Table 2 showed that the knee joint mobility of patients in the 2 groups gradually increased with time and reached a peak 12 weeks after surgery $(\mathrm{P}<0.05)$, however, there was no significant difference in knee joint mobility between the 2 groups at each time point after surgery $(\mathrm{P}>0.05)$. The excellent and good rates of the 2 groups were both above $80 \%$, indicating that the therapeutic effects of the 2 surgical options were similar. In addition, because arthroscopic angioplasty can eliminate the horizontal shear force while preserving the load transmission function of the meniscus, the current biomechanics of the knee joint are similar to the normal structure (11). Therefore, it is clinically believed that plastic surgery should be performed in most cases when the patient's condition permits. If plastic surgery is not effective, it is necessary to retain relatively stable and healthy meniscus tissue as much as possible (12).

Postoperative pain is an important factor affecting functional recovery of the knee joint. Therefore, this study analyzed 71 patients with discoid meniscus injury after arthroscopic treatment. The results showed that $15.49 \%$ 
Table 3 Single factor analysis of postoperative pain in patients with discoid meniscus injury of knee joint

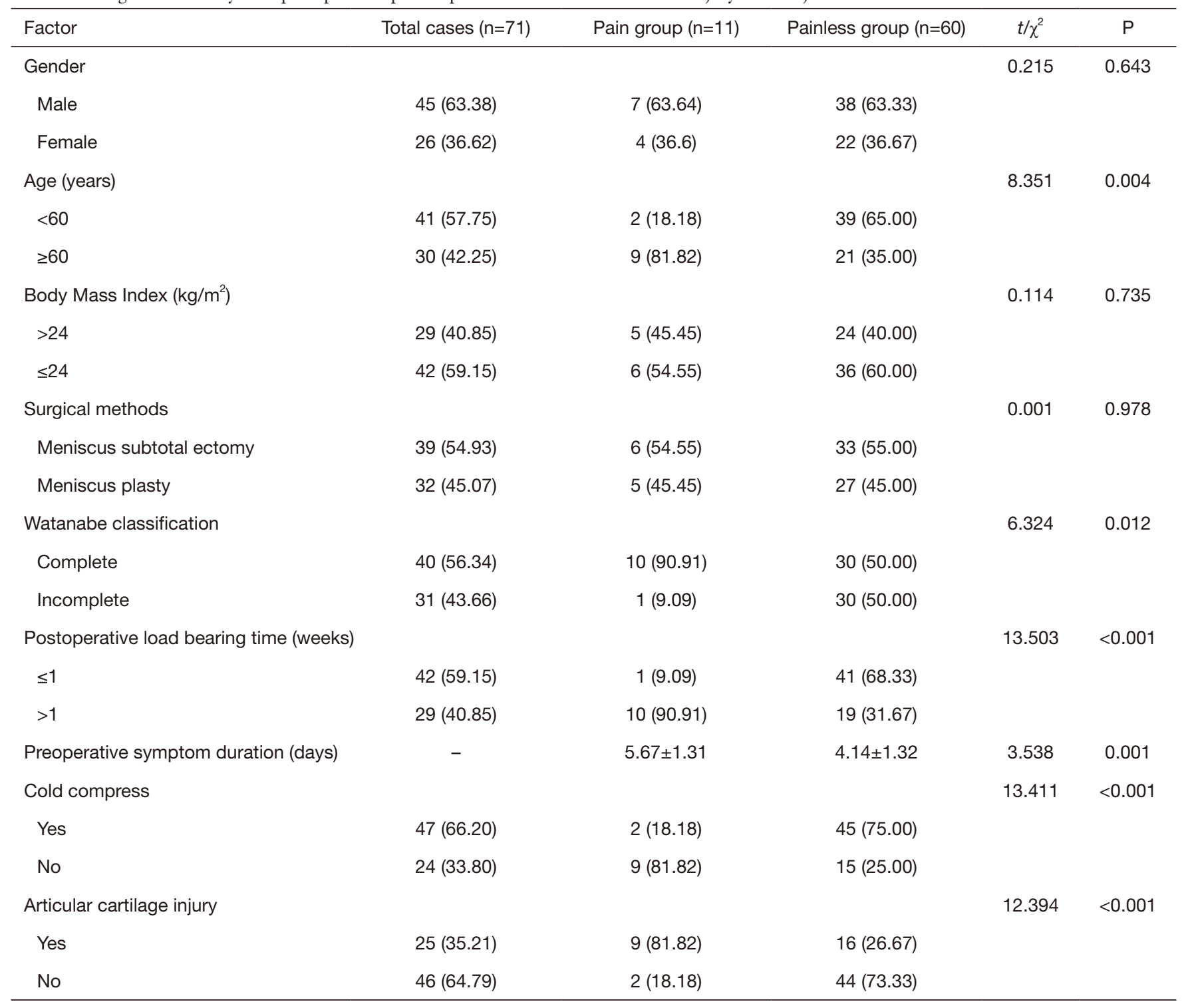

Table 4 Multi-factor analysis of postoperative pain in patients with discoid meniscus injury of the knee joint

\begin{tabular}{lccccc}
\hline Factor & Regression coefficient & SEM & Wald $\chi^{2}$ & $\mathrm{P}$ & OR (95\% Cl) \\
\hline Age ( $\leq 60$ vs. $>60$ years) & 0.761 & 0.164 & 4.531 & 0.004 & $2.14(1.55-2.95)$ \\
Watanabe classification (complete vs. incomplete) & 0.759 & 0.776 & 4.353 & 0.731 & $2.14(0.47-9.78)$ \\
Postoperative load bearing time ( $\leq 1$ vs. $>1$ week) & 0.734 & 0.131 & 4.458 & $<0.001$ & $2.08(1.61-2.69)$ \\
Preoperative symptom duration & 0.891 & 0.146 & 4.384 & $<0.001$ & $2.44(1.83-3.25)$ \\
Cold compress (yes vs. no) & 0.795 & 0.114 & 4.838 & $<0.001$ & $2.21(1.77-2.77)$ \\
Articular cartilage injury (yes vs. no) & 0.747 & 0.231 & 4.445 & $<0.001$ & $2.11(1.34-3.32)$ \\
\hline
\end{tabular}

SEM, standard error of mean; OR, odd ratio; $\mathrm{Cl}$, confidence interval. 
$(11 / 71)$ of patients had postoperative pain. Multivariate logistic regression analysis was used to analyze related highrisk factors of postoperative pain. Results in Table 4 showed that age, preoperative symptom duration, postoperative weight-bearing time $\leq 1$ week, cold compress, and articular cartilage injury were all independent risk factors that affected postoperative pain in patients with discoid meniscus injury $(\mathrm{P}<0.05)$. This might be due to the following reasons: (I) elderly patients have a higher incidence of developing degenerative lesions in the knee joint tissues, and blood circulation is not as good as younger patients; (II) a longer duration of symptoms before surgery indicates more severe joint damage; (III) premature weight bearing after surgery could increase pressure on the knee joint, which might affect the prognosis of the incision, and delay the recovery of joint function; (IV) postoperative cold compresses can promote local vasoconstriction, control the bleeding of small blood vessels, and reduce the sensitivity of nerve endings, thereby reducing swelling and pain; (V) tissue damage of articular cartilage increases the risk of knee joint injury (13-15). Therefore, patients with discoid meniscus injury (especially elderly patients) should receive arthroscopic surgery at an early stage, cold compresses should be applied after surgery, and the weight-bearing time should be delayed to promote the recovery of knee joint function (16).

In addition, clinical practice shows that arthroscopic surgery still has complications such as broken intra-articular instruments, injury of normal intra-articular tissues, injury of tissues around the entrance of arthroscopy, extravasation of washing fluid, intraarticular hematoma, infection, phlebitis, nerve injury and so on. Therefore, in the process of clinical treatment, it is necessary to constantly improve the operator's operating skills, reduce intraoperative injuries, strengthen postoperative intervention and rehabilitation of patients, and prevent the occurrence of infection and secondary injuries.

In summary, arthroscopic treatment demonstrated efficacy for patients with discoid meniscus injury of the knee joint. Factors such as the age of the patient, the duration of preoperative symptoms, articular cartilage damage, postoperative cold compresses, and postoperative weight-bearing time were all independent risk factors for postoperative pain. Therefore, effective prevention and control measures should be taken for patients at high risk of postoperative pain to avoid or relieve the postoperative pain and promote the recovery of knee joint function. The shortcomings of this study are that the sample size was small and the follow-up time was short. Therefore, future clinical studies with a larger study population and longer followup duration are warranted in order to confirm the efficacy of arthroscopy for the treatment of discoid meniscus injury, and the sample size can be expanded and the follow-up time might be increased in the future.

\section{Acknowledgments}

Funding: None.

\section{Footnote}

Reporting Checklist: The authors have completed the STROBE reporting checklist. Available at http://dx.doi. org/10.21037/apm-20-1899.

Data Sharing Statement: Available at http://dx.doi. org/10.21037/apm-20-1899.

Conflicts of Interest: All authors have completed the ICMJE uniform disclosure form (available at http://dx.doi. org/10.21037/apm-20-1899). The authors have no conflicts of interest to declare.

Ethical Statement: The authors are accountable for all aspects of the work in ensuring that questions related to the accuracy or integrity of any part of the work are appropriately investigated and resolved. This study was approved by the ethics committee of Luwan Branch of Ruijin Hospital affiliated to Shanghai Jiao Tong University Medical College (No. 2019-129). All patients provided informed consent. All procedures performed in this study involving human participants were in accordance with the Declaration of Helsinki (as revised in 2013).

Open Access Statement: This is an Open Access article distributed in accordance with the Creative Commons Attribution-NonCommercial-NoDerivs 4.0 International License (CC BY-NC-ND 4.0), which permits the noncommercial replication and distribution of the article with the strict proviso that no changes or edits are made and the original work is properly cited (including links to both the formal publication through the relevant DOI and the license). See: https://creativecommons.org/licenses/by-nc-nd/4.0/.

\section{References}

1 Rai MF, Brophy RH, Sandell LJ. Osteoarthritis following 
meniscus and ligament injury: insights from translational studies and animal models. Curr Opin Rheumatol 2019;31:70-9.

2 Restrepo R, Weisberg MD, Pevsner R, et al. Discoid Meniscus in the Pediatric Population: Emphasis on MR Imaging Signs of Instability. Magn Reson Imaging Clin N Am 2019;27:323-39.

3 Zhou Z, Xiao L, He C, et al. Application of assisted portal under anterior horn of lateral meniscus for the treatment of discoid meniscus injury. Knee 2019;26:1125-35.

4 Ohnishi Y, Nakashima H, Suzuki H, et al. Arthroscopic treatment for symptomatic lateral discoid meniscus: The effects of different ages, groups and procedures on surgical outcomes. Knee 2018;25:1083-90.

5 Han SB, Babu CP, Choi JH, et al. Regeneration of lateral discoid meniscus after arthroscopic partial meniscectomy in an adult patient. Knee Surg Sports Traumatol Arthrosc 2018;26:2278-81.

6 Choi YH, Seo YJ, Ha JM, et al. Collagenous Ultrastructure of the Discoid Meniscus: A Transmission Electron Microscopy Study. Am J Sports Med 2017;45:598-603.

7 Atay OA, Pekmezci M, Doral MN, et al. Discoid meniscus: an ultrastructural study with transmission electron microscopy. Am J Sports Med 2007;35:475-8.

8 Kim SH, Ahn J, Kim TW, et al. Midbody of the medial meniscus as a reference of preservation in partial meniscectomy for complete discoid lateral meniscus. Knee Surg Sports Traumatol Arthrosc 2019;27:2558-67.

$9 \mathrm{Ng}$ YH, Tan SHS, Lim AKS, et al. Meniscoplasty leads to good mid-term to long-term outcomes for children and adolescents with discoid lateral meniscus. Knee Surg

Cite this article as: $\mathrm{Lu} \mathrm{J}$, Chen Y, Hu M, Sun C. Clinical efficacy of arthroscopy in the treatment of discoid meniscus injury and related risk factors for postoperative pain. Ann Palliat Med 2020;9(6):4002-4009. doi: 10.21037/apm-20-1899
Sports Traumatol Arthrosc 2020. [Epub ahead of print].

10 Haskel JD, Uppstrom TJ, Dare DM, et al. Decline in clinical scores at long-term follow-up of arthroscopically treated discoid lateral meniscus in children. Knee Surg Sports Traumatol Arthrosc 2018;26:2906-11.

11 Yang X, Shao D. Bilateral discoid medial Meniscus: Two case reports. Medicine 2019;98:e15182.

12 Johnson B, Heaver C, Gilbert R, et al. Anterior meniscopexy: a meniscal sparing technique for the treatment of locking but intact discoid lateral meniscus. Knee Surg Sports Traumatol Arthrosc 2018;26:1158-63.

13 Griffin DW, Kinnard MJ, Formby PM, et al. Outcomes of Hip Arthroscopy in the Older Adult: A Systematic Review of the Literature. Am J Sports Med 2017;45:1928-36.

14 Tennent DJ, Hylden CM, Johnson AE, et al. Blood Flow Restriction Training After Knee Arthroscopy: A Randomized Controlled Pilot Study. Clin J Sport Med 2017;27:245-52.

15 Li J, Li ZL, Zhang H, et al. Long-term Outcome of Multiple Small-diameter Drilling Decompression Combined with Hip Arthroscopy versus Drilling Alone for Early Avascular Necrosis of the Femoral Head. Chin Med J (Engl) 2017;130:1435-40.

16 Hamrin Senorski E, Samuelsson K, Thomeé C, et al. Return to knee-strenuous sport after anterior cruciate ligament reconstruction: a report from a rehabilitation outcome registry of patient characteristics. Knee Surg Sports Traumatol Arthrosc 2017;25:1364-74.

(English Language Editor: C. Betlazar-Maseh) 